\title{
BIOLOGIA REPRODUTIVA DE JUMENTOS. II. CARACTERÍSTICAS FÍSICAS E MORFOLÓGICAS DO SEMMEN *
}

\author{
REPRODUCTIVE BIOLOGY OF IONKEY. II. PHYSYCAL AND MORFHOLOGYCAL CHARACTERISTICS OF SEMEN
}

Rosana Nogueira de MORAIS'; Raul Gastão MUCCIOL,O'; Wilson (ionçalves VIANA"

\begin{abstract}
RESUMO
Foram estudadas as características físicas e morfológicas do sêmen de seis (06) jumentos da rą̧a Pêga. com idade variando entre 3 e 9 anos. As colheitas de sêmen foram realizadas durante a estaçĩo de monta, numa frequêencia de 2 cjaculações por semana, totalizando setenta (70) amostras. Os valores médios encontrados para os parâmetros espermáticos foram os seguintes: volume total $(59.88 \pm 15.54 \mathrm{ml})$, volume de $\mathrm{gcl}(8.19 \pm 4,07 \mathrm{ml})$, motilidade espermática total $(81.93 \pm 6.27 \%)$. motilidade progressiva $(72.86 \pm 7,20 \%)$, vigor espermático $(4,63 \pm 0.49)$, concentração espermática $\left(444.11 \pm 182,72 \times 10 \% / \mathrm{mm}^{3}\right)$. total de espermatozóides por ejaculado $(24.88 \pm 9.59 \times 109)$. total de defeitos espermáticos $(15,60 \pm 3,62 \%)$, defeitos maiores $(8,55$ $\pm 2,14 \%$ ) e defeitos menores $(7,04 \pm 2,56 \%$ ). Essas médias não diferem muito daquelas reportadas para asininos. No entanto. ao serem comparadas com dados relativos a equinos. observa-se a existência de diferenças em alguns aspectos. O volume total do cjaculado de jumentos é inferior. porém com menor incidência e volume de gel. portanto, a porção rica em espermatozóides apresenta praticamente o mesmo volume. Entretanto, a concentraçāo espermátică e o número total de espermatozóides são maiores do que em garanhōes. Além disso. pela avaliação da motilidade e vigor espermáticos. constata-se que os jumentos apresentam. de um modo geral, ejaculados de melhor qualidade. Em programas de inseminaçāo artificial estas características indicam a possibilidade de maior número de doses por ejaculado e melhores índices de congelabilidade.
\end{abstract}

LNITERMOS: Reprodução: Jumentos; Sêmen: Morfologia

\section{INTRODUÇÃO}

A avaliação do sêmen e determinação de parâmetros espermáticos é etapa indispensável dentro do exame de animais a serem utilizados como doadores de sêmen em programas especiais de reprodução. Serve de base para selaçāo e estimativa do potencial reprodutivo de cada animal, incluindo grau de congelabilidade de amostras e proporção doses por amostra em programas de inseminação artificial. Em jumentos, as informações obtidas são bastante limitadas e toma-se geralmente como referência os dados pertinentes a equinos. Deste modo, o nosso objetivo é estudar as características físicas e morfológicas do sêmen de jumentos, durante a estação de monta, fazendo sempre que relevante, uma comparação com os dados publicados para equinos.

Considerando-se o volume do ejaculado, tanto de asininos como de outras espécies animais, encontramos uma grande variabilidade, uma vez que esta característica seminal sofre efeito de vários fatores tais como raça, idade, freqüência de cjaculação, duração do tempo de excitação, entre outros. Os valores encontrados para o volume médio do ejaculado de asininos variam desde $37,0 \mathrm{ml}^{30}$ até $147 \mathrm{ml}^{7}$, porém a maioria dos resultados encontram-se numa faixa entre 40 e 60 $\mathrm{ml}^{1,15,18,20,22,24,28,42}$. A ocorrência da fração gelatinosa no ejaculado de jumentos é também variável. Em alguns estudos não foj detectada $\mathrm{cm}$ nenhum animal ${ }^{22.30}$, enquanto que $\mathrm{em}$ outros, ficou limitada à estação de montai ${ }^{21520.28}$, sendo registrados volumes relativamente baixos ${ }^{12}$.

De um modo geral, o aspecto do sêmen de asininos é mais turvo e mais leitoso do que o sêmen de equinos, variando entre leite-aquoso e leite cremoso, com cor oscilando entre branco-acinzentado e branco amarelado ${ }^{3,20.28}$.

Na avaliação da motilidade espermática, os valores médios

1-Professor Assistente - Universidade fiederal du Paranai

2-Professor Associado - Fiaculdade de Medicina Veterinárial e \%ontecnia da USP'

3-Professor Doutor - Faculdade de Medicina Veterinárial e Zootecnial da USP

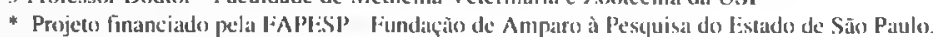


MORAIS, R.N.: MUCCIOLO. R.G.: VIANA. W.G. Biologia reprodutiva de jumentos. II. Características físicals e morfológicas do sèmen. Braz.. J. vet. Kes. anim. Sci.., Sao Paulo. v. 31, n. I. P. 49-57, 1994

encontrados para motilidade total estão entre 60 e $100 \%$, sendo que a maioria dos autores obteve resultados superiores a $80 \% 1,15,28,30$. Ao analisarmos também o tipo de movimento execulado pelos espermatozóides, especificamente a porcentagem de células com movimento progressivo, encontramos médias entre 40 e $85 \%^{2,15,18,20,21.22}$. Um parâmetro que complementa os dados de motilidade é a determinação do vigor espermático, porém, foi analisado por um número bastante restrito de autores. OCES et al. ${ }^{30}$, encontraram uma médial de 4,2 em 60 ejaculados analisados, praticamente o mesmo publicado por HENRY el al. ${ }^{18}$.

A concentração espermática e o número total de espermatozóides, assim como outros parâmetros do ejaculado de asininos, são muito variáveis. Os valores médios apresentados variam desde $224 \times 10^{9} / \mathrm{mm}^{3}$ e um total de $6,90 \times 10^{4}$ espermatozóides ${ }^{18}$ até concentrações elevadas como $665 \mathrm{x}$ $10 \% / \mathrm{mm}^{3}$, ou um número total de espermatozóides da ordem de 26 bilhões de células 22 .

Ao se estudar a morfologia espermática de equídeos, o primeiro ponto de desacordo está na classificação e avaliação do real impacto de determinados níveis de patologia espermálica sobre a capacidade reprodutiva dos animais estudados. Alguns autores adotam a classificação de BLOM

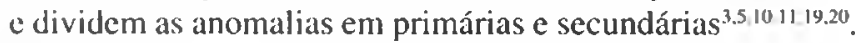
Outros preferem classificar as anomalias de acordo com sua localização - cabeça,peça intermediária e cauda - ${ }^{27,37,40}$ ou, ainda, utilizam a nova classificação proposta por BLOM", dividindo os defeitos em maiores e menores ${ }^{1,14.18 .31}$. NISHIKAWA et al. ${ }^{29}$ descrevem algumas diferenças entre equinos e asininos quanto à morfologia espermática. O comprimento total dos espermatozóides de jumentos é significativamente maior, com formato de cabeça mais arredondado. As considerações acerca da patologia espermática são basicamente as mesmas feitas para equinos. As alterações mais freqüentes no sêmen de garanhões com fertilidade normal incluem gotas citoplasmáticas proximais e distais, caudas cnroladas e dobradas, implantação abaxial e, em menor número, alterações de cabeça. Não se sabe ainda se cssas alterações são realmente indicativas de alguma disfunção, sendo que a implantação abaxial é considerada normal por vários autores ${ }^{311,37}$, assim como uma certa variação no tamanho das cabeças espermáticas, com aparecimento de cabeças pequenas normais ou com assimetria unilateral ${ }^{6,10.11}$.

No entanto, um equino de boa fertilidade deve, além de normalidade no comportamentoo sexual, na concentração e motilidade espermáticas, apresentar $80 \%$ de espermatozóides normais, menos do que $10 \%$ de gotas citoplasmáticas, $3 \%$ de caudas dobradas e enroladas, $3 \%$ de cabeças isoladas e $1 \%$ de outras formas anormais ${ }^{6}$. Para outro autor ${ }^{25}$ o limite permissível para o ejaculado equino seria um total de 25 a $30 \%$ de patologias sendo até $10 \%$ de defeitos maiores e até $20 \%$ de defeitos menores.

Em estudos realizados com jumentos, os níveis de patologia espermática oscilam entre 6 e $14 \%$ no total $\left.\right|^{1,1+18,20,21,24}$. Dentre as anomalias encontradas parece haver um predomínio de caudas enroladas e dobradas, com baixa incidência de gotas citoplasmáticas ${ }^{20.29}$

\section{MATERIAI, E MÉTODO}

\section{ANIMAIS}

Foram utilizados seis (06) jumentos da raça Pêga, pertencentes a três propricdades rurais do município de São CarlosS.P., os quais foram designados por letras de A a F. A altura e peso médio foram de $1,30 \mathrm{~m} \mathrm{e} 400 \mathrm{~kg}$, respectivamente, c a idade de cada um no inicio do experimento era de $4(A)$, 6(B), 6(C), 8(D), 9 (E) e 3(F) anos. Todos foram submelidos previamente a exame clínico $\mathrm{c}$ andrológico, tendo sido considerados aptos para o experimento. Durante todo o período experimental, de outubro de 1988 a abril de 1989, os animais foram mantidos em piquetes individuais, com livre acesso a baias, recebendo ração e suplementação mineral balanceadas, com água à vontade.

\section{COLHEITA DE SÊMEN}

As colheitas foram realizadas de acordo com a técnica utilizada para garanhões ${ }^{35}$, através de vagina artificial. Como manequins foram utilizadas éguas $\mathrm{cm}$ cio natural $\mathrm{c}$, apenas para o jumento $C$, jumentas em cio natural, por se tratar de um animal não habituado a realizar coberturas de éguas. Foram realizadas duas colheitas por semana, totalizando 70 ejaculados.

\section{AVALIAÇÃo DO SÊMEN}

Após colheita o sêmen foi levado imediatamente ao laboratório para ser analisado macro e microscopicamente e os resultados foram anotados em fichas individuais.

Toda a vidraria utilizada para a realização dos exames foi préaquecida a $35^{\circ} \mathrm{C}$ antes de ser colocada em contato com o sêmen, evitando-se a ocorrência de choques térmicos.

\section{Volume, aspecto e cor}

O sêmen foi transferido lentamente para uma proveta graduada $(100$ ou $200 \mathrm{ml})$ para a avaliação do volume total do ejaculado, expresso em ml. Na presença da fração gelatinosa, o sêmen foi filtrado $\mathrm{cm}$ gaze estéril para a determinação do volume de $\mathrm{gel}(\mathrm{ml})$. O aspecto e a cor também foram observados e registrados. 


\section{Motilidade e vigor}

A porcentagem de espermatozóides móveis loi determinada imeoiatamente após a colleita, colocando-se uma gota de sêmen (livre de gel) entre lâmina e lamínula. A motilidade foi estimada subjetivamente através da avaliação visual em microscópio óptico comum* equipado com platina aquecida** a $35^{\circ} \mathrm{C}$, num aumento de 400 vezes. $O$ resultado, apresentado sob a forma de porcentagem média de espermatozóides com movimento total e com movimento progressivo, foi registrado após a observação de pelo menos 5 campos microscópicos, em duas preparações diferentes do mesmo ejaculado.

O vigor espermático foi classificado de acordo com WALTON", dentro da escala de 0 (vigor de movimento nulo) até 5 (vigor de nfovimento máximo).

\section{Concentração espermática}

Para a determinação da concentração de espermatozóides na fração livre de gel de cada ejaculado, foi feita uma diluição do sêmen a $1: 100$, com auxílio de pipeta de Sahli, em solução de formol salino tamponada. () número de espermatozóides foi determinado através da contagem em câmara de Neubauer melhorada e registrado em número de células espermáticas $\mathrm{x}$ $10^{4}$ por $\mathrm{mm}^{3}$.

\section{Número total de espermatozóides}

Através da multiplicação do valor do volume do ejaculado. livre de gel, èm ml. pelo número de espermatozóides por ml, calculou-se o número total de espermatozóides do ejaculado, expresso em unidade de bilhões de células espermáticas.

\section{Morfologia espermática}

Para avaliação da morfologia espermática foi preparada uma amostra de cada cjaculado, colocando-se 3 a 4 gotas de sêmen, dependendo da concentração do mesmo, em um tubo contendo $2 \mathrm{ml}$ de solução de formol salina tamponada. Lima gota desta amostra foi colocada entre lâmina e lamínula, numa preparação úmidath. e examinada sob imersão, num aumento de 1,250 vezes em microscópio de contraste de interlerência diferencial*, segundo DOTT ${ }^{10}$ e HARAZYMOWYCZ et al. ${ }^{17}$. Foram contados 400 espermatozóides para determinar-se a porcentagem de células normais e células com anomalias de acrossomo, cabeça, peça intermediária ou de cauda e, ainda, a incidência de células decaptadas ou com gotas citoplasmáticas. A classificação das lormas espermáticas foi baseada, principalmente, nos trabalhos de BIELANSKI ${ }^{34}$, DOTT ${ }^{10}$ e BIELANSKI et al. ${ }^{6}$ e os resultados foram agrupados em defeitos maiores e menores, segundo BLOM".

\section{RESULTADOS}

\section{VOLUME, ASPECTO E COR}

Os resultados médios obtidos para o volume do ejaculado estão demonstrados na Tab.1. O coeficiente de variação global foi de $26 \%$, sendo detectada a presença da fração gelatinosa em 16 dos mesmos, numa frequiência de $22,85 \%$ e volume médio de $8,19 \pm 4,07 \mathrm{ml}$.

Quanto ao aspecto e cor das amostras houve uma variação entre leite-aquoso e leite-cremoso e predomínio da cor branca-acinzentada ou branca-amarelada, com variações muito discretas.

\section{MOTILIDADE E VIGOR}

Os resultados encontrados para a porcentagem de motilidade total e progressiva estão apresentados na Tab.2. A média global obtida para o vigor espermático foi de 4,63 $\pm 0,49$. sendo que três dos jumentos (A, B e E) tiveram todas suas amostras classificadas com valor máximo $(5,0)$. O coeficiente de variação para motilidade total foi de $8 \%$ e para motilidade progressiva e vigor de $10 \%$.

\section{CONCENTRAÇÃO E NÚMERO TOTAL DE ESPERMA- TOZÓIDES}

A Tab. 3 contém os resultados médios obtidos para a concentração espermática por $\mathrm{mm}^{3}$ e o número total de espermatozóides, para cada animal e para o total de amostras. Os coeficientes de variação para a concentração espermática e total de espermatozóides foram, respectivamente, $41 \%$ e $38 \%$.

\section{MORFOLOGIA ESPERMÁTICA}

Na Tab.4 são apresentados os valores médios da porcentagem de deleitos maiores e menores, bem como o total de anomalias espermáticas. Os coeficientes de variação foram $25 \%$ e 2.3\% para média de defeitos maiores e total de patologia, respectivamente, sendo maior para a média de defeitos meno$\operatorname{res}(36 \%)$.

Os resultados médios individuais para a porcentagem de defeitos de acrossomo, cabeça, peça intermediária e cauda, além da incidência média de cabeças isoladas e gotas citoplasmáticas, estão contidos na Tab.5.

\footnotetext{
" Microscópio óptico ME*OTTA - Tehecoslovíquia

* Eletrovet - Sáio Paulo - Sl?
} 
MORAIS, R.N.; MUCCIOLO, R.(i., VIA.VA. W.G. Biolengia reprodutiva de jumentos. II. Características físicas e morfológicas do sémen. Braz.. J. vet. Res. a nim. Sci., Săo Paulo, v. 31, n. I. p. .99-57, 1994.

\section{TABELA 1}

Média $(\bar{X})$ e Desvio Padrão (DP) do volume total do ejaculado (ml) de jumentos, incluindo os valores máximos e mínimos. São Carlos-SP, $1988 / 89$.

\begin{tabular}{cccccc}
\hline JUMENTOS & $\mathrm{N}^{2}$ & $\mathrm{X}$ & $\mathrm{DP}$ & MÁXIMO & MÍNIMO \\
\hline A & 14 & $64,86^{+}$ & 16,48 & 105 & 40 \\
B & 14 & $68,43^{\mathrm{a}}$ & 14,94 & 96 & 45 \\
C & 15 & $50,40^{\mathrm{b}}$ & 9,01 & 68 & 38 \\
D & 6 & $53,33^{\mathrm{b}}$ & 11,29 & 75 & 45 \\
E & 11 & $50,00^{\mathrm{h}}$ & 13,32 & 66 & 31 \\
F & 10 & $70.00^{\mathrm{h}}$ & 13,36 & 92 & 53 \\
\hline Total & 70 & 59,88 & 15,54 & 105 & 31 \\
\hline
\end{tabular}

$\mathrm{N}^{2}=$ Número de ejaculados

${ }^{2 b}=$ Médias com diferentes letras diferem significativamente $(\alpha=0,05)$

TABELA 2

Média $(\bar{X})$ e Desvio Padrão (DP) da motilidade espermática total (\%) e motilidade espermática progressiva (\%) do ejaculado de jumentos, incluindo valores máximos e mínimos ( ). São Carlos-SP, $1988 / 89$.

\begin{tabular}{|c|c|c|c|c|c|}
\hline \multirow{2}{*}{$\begin{array}{c}\text { JUMENTOS } \\
\mathrm{A}\end{array}$} & \multirow{2}{*}{$\frac{N^{\circ}}{14}$} & \multicolumn{2}{|c|}{$\begin{array}{l}\text { MOTILIDADE: TOTAL } \\
(\bar{X} \pm D P)\end{array}$} & \multicolumn{2}{|c|}{$\begin{array}{l}\text { MOTILIDADE PROGRESSIVA } \\
(\overline{\mathrm{X}} \pm \mathrm{DP})\end{array}$} \\
\hline & & $81.76 \pm 5.41^{\mathrm{b}}$ & $(70)-90)$ & $73.21 \pm 5.41^{\mathrm{b}}$ & $(65-80)$ \\
\hline B & 14 & $81.79 \pm 6,39^{10}$ & $(70-90)$ & $74.29 \pm 5.13^{\mathrm{b}}$ & $(65-80)$ \\
\hline C & 15 & $82.67 \pm 4.17^{b}$ & $(80-90)$ & $74.33 \pm 4.95^{\mathrm{b}}$ & $(65-80)$ \\
\hline D & 06 & $68.33 \pm 4.08$ & $(60-70)$ & $6\left(0,83 \pm 5,84^{c}\right.$ & $(50-65)$ \\
\hline $\mathrm{E}$ & 11 & $86,36 \pm 2,34$ & $(85-90)$ & $80,95 \pm 3,50^{\prime \prime}$ & $(75-85)$ \\
\hline $\mathrm{F}$ & 10 & $84.50 \pm 1.58^{\mathrm{b}}$ & $(80-85)$ & $67.00 \pm 5,86^{\circ}$ & $(55-75)$ \\
\hline TOTAL & 70 & $81,93 \pm 6,27$ & $(60-90)$ & $72,86 \pm 7,20$ & $(50-85)$ \\
\hline
\end{tabular}

$\mathrm{N}^{2}=$ Número de ejaculados

${ }^{a}$ abc $=$ Médias com diferentes letras diferem significativamente $(\alpha=0,05)$ 


\section{TABELA 3}

Média $(\bar{X})$ e Desvio Padrão (DP) da concentração espermática $\left(x 10^{3} / \mathrm{mm}^{3}\right)$ e do número total de espermatozóides $\left(x 10^{4}\right)$ do ejaculado de jumentos, incluindo valores máximos e mínimos ( ). São Carlos-SP, 1988-89.

\begin{tabular}{|c|c|c|c|c|c|}
\hline \multirow{2}{*}{$\frac{\text { JUMENTOS }}{\wedge}$} & \multirow{2}{*}{$\frac{N^{N}=}{14}$} & \multicolumn{2}{|c|}{$\begin{array}{c}\text { CONCENTRAÇÃO } \\
\text { ESPERMÁTICA } \\
(\bar{X} \pm \text { DP })\end{array}$} & \multicolumn{2}{|c|}{$\begin{array}{l}\text { TOTAL DE ESPERMATOZÓIDES } \\
\qquad(\overline{\mathrm{X}} \pm \mathrm{DP})\end{array}$} \\
\hline & & $284.82 \pm 88.82^{4}$ & $(170.475)$ & $17.43 \pm 6,\left(12^{\triangleleft}\right.$ & $(8,00-29,33)$ \\
\hline B & 14 & $405.71 \pm 94,38^{\prime \prime}$ & $(270-615)$ & $27.31 \pm 6,84^{\prime \prime}$ & $(16,47-38,88)$ \\
\hline C & 15 & $429,33 \pm 122,29^{\mathrm{h}}$ & $(205-690)$ & $20,26 \pm 6.55^{\mathrm{cll}}$ & $(9,84-26,27)$ \\
\hline D & 6 & $504,17 \pm 145,86^{\mathrm{n}}$ & $(330-705)$ & $26.87 \pm 9,\left(02^{1 \kappa}\right.$ & $(16,2-36,75)$ \\
\hline E & 11 & $743.18 \pm 180,42$ & $(395-905)$ & $36,21 \pm 11,79$ & $(20,31-51,81)$ \\
\hline i: & 10 & $378,00 \pm 41,43^{\circ}$ & $(310-455)$ & $25.23 \pm 5.34^{\mathrm{bc}}$ & $(19.45-33,86)$ \\
\hline TOTAL & 70 & $444.11 \quad \pm 182.72$ & $(170-905)$ & $24,88 \pm 9,59$ & $(8,00)-51,81)$ \\
\hline
\end{tabular}

№ $\quad$ Número de cjaculados

atheat = Médias com diferentes letras difcrem significativamente $(\alpha=0,05)$

\section{TABELA 4}

Média $(\bar{X})$ e Desvio Padrão (DP) da porcentagem de defeitos maiores e menores e total de anomalias espermáticas (\%) no sêmen de jumentos. São Carlos-SP. 1988/89.

\begin{tabular}{|c|c|c|c|c|c|}
\hline & & DEFEITOS MAIORIS & DEFEITOS MENORES & $\underline{\mathrm{TO}^{-}}$ & TAL \\
\hline JUMENTOS & № & $\bar{X} \pm D P$ & $\bar{X} \pm D P$ & $\bar{x}$ & $\pm D P$ \\
\hline A & 14 & $9.41 \pm 1.62$ & $8.38 \pm 1.41$ & 17,18 & $\pm 1,18^{\mathrm{b}}$ \\
\hline B & 14 & $8,39 \pm 2,00$ & $7,95 \pm 1.71$ & 16.34 & $\pm 2.81^{\mathrm{h}}$ \\
\hline C & 15 & $7.13 \pm 1,36$ & $5,27 \pm 1,17$ & 12,40 & $\pm 1,90^{\circ}$ \\
\hline D & 6 & $9,12 \pm 0,80$ & $11,67 \pm 2,96$ & 20.79 & $\pm 2,58^{4}$ \\
\hline E & 11 & $7.34 \pm 2.82$ & $4.86 \pm 2.10$ & 12,20 & $\pm 3,62^{\circ}$ \\
\hline $\mathrm{F}$ & 10 & $10,70 \pm 1,36$ & $6,20 \pm 1,08$ & 16,90 & $\pm 1,98^{\mathrm{h}}$ \\
\hline TOTAL & 70 & $8,55 \pm 2,14$ & $7,04 \pm 2,56$ & 15,60 & $\pm 3,62$ \\
\hline
\end{tabular}

№ = Número de ejaculados

ab = Médias com diferentes letras diferem signilicativamente $(\alpha=0,05)$ 
MORAIS, R.N.: MLCCIOL.O, R.G.; VIA.VA, W.G. Biologia reproduliva de jumentos. Il. Características físicas e morfológicas do sêmen. Braz. J. vet. Res. anim. Sci., Săı Paulo. v. 31, n.I. p. $49-57,1944$

\section{TABELA 5}

Média $(\bar{x}$ ) e desvio padrão DP da porcentagem de anomalias espermáticas, segundo a localização, no sêmen de jumentos. São Carlos-SP, 1988/99.

\begin{tabular}{|c|c|c|c|c|c|c|c|}
\hline CARACTI:RIS & $\begin{array}{c}A \\
(14) \\
\bar{X} \pm D P\end{array}$ & $\begin{array}{c}\mathrm{B} \\
(14) \\
\bar{X} \pm \mathrm{DP}\end{array}$ & $\begin{array}{c}\mathrm{C} \\
(15) \\
\bar{X} \pm \mathrm{DP}\end{array}$ & $\begin{array}{c}D \\
(06) \\
\bar{X} \pm D P\end{array}$ & $\begin{array}{l}E \\
(11) \\
\bar{X} \pm D P\end{array}$ & $\begin{array}{c}F \\
(10) \\
\bar{X} \pm D P\end{array}$ & $\begin{array}{l}\text { TOTAL } \\
(70) \\
\bar{x} \pm D P\end{array}$ \\
\hline Acrossomo & $1,96 \pm 0,66$ & $1,18 \pm 0,33$ & $1,98 \pm 0,54$ & $0,79 \pm 0,78$ & $1,20 \pm 0,83$ & $1,80 \pm 0.86$ & $1.48 \pm 0.50$ \\
\hline Cabeça & $1,25 \pm 0,69$ & $1,95 \pm 1,10$ & $3,55 \pm 1,07$ & $4,04 \pm 1,47$ & $4,07 \pm 1,04$ & $6.05 \pm 1,48$ & $3.48 \pm 1,71$ \\
\hline Peça Interm. & $2.61 \pm 1,32$ & $1,25 \pm 0,84$ & $1.60 \pm 0.67$ & $1,75 \pm 0,35$ & $1,89 \pm 0,77$ & $2,45 \pm 0,60$ & $1,92 \pm 0.52$ \\
\hline Cauda & $3,98 \pm 1,04$ & $4,73 \pm 1,86$ & $2,62 \pm 1,13$ & $8,33 \pm 2,22$ & $2,48 \pm 2,06$ & $3,05 \pm 1,44$ & $4,20 \pm 2,20$ \\
\hline Cabeça Isolada & $0.27 \pm 0.53$ & $0.25 \pm 0,29$ & $0.58 \pm 0,37$ & $0.88 \pm 0.26$ & $0,52 \pm 0,48$ & $0,75 \pm 0,59$ & $0.54 \pm 0.25$ \\
\hline Gota Proximal & $2,45 \pm 1,18$ & $2,61 \pm 0,86$ & $1,18 \pm 0,59$ & $1,29 \pm 0,46$ & $0,64 \pm 0,81$ & $0,45 \pm 0,28$ & $1,43 \pm 0,90$ \\
\hline Gota Distal & $5,30 \pm 1,28$ & $4,34 \pm 1,20$ & $0,88 \pm 0,66$ & $3,71 \pm 1,87$ & $1,41 \pm 2,30$ & $2,35 \pm 0,97$ & $3,00 \pm 1,73$ \\
\hline
\end{tabular}

Obs. ()s números entre parênteses representam o número de cjaculados analisados.

\section{DISCUSSÃO E CONCIUSÕES}

O aspecto das amostras de sêmen por nós analisadas coincide com observações prévias de outros autores ${ }^{7.20 .28}$. Com relação ao volume do ejaculado, a média obtida no presente experimento foi superior aos valores apresentados para jumentos de porte semelhante $18,20,22,28,30$ ou bastante semelhantes aos dados de outros autores ${ }^{15.24 .42}$. É difícil uma discussão mais ampla. uma vez que esta característica do ejaculado sofre efeito de vários fatores tais como idade, raça, época do ano e frequiencia de cjaculação, entre outras. No entanto, ao se comparar os nossos resultados com aqueles obtidos para garanhōes $11.26,31,3,3$, observa-se que o volume do ejaculado de jumentos é realmente inferior ao de equinos. Porém, ao se considerar a porcentagem da fração gelatinosa presente no cjaculado, o que se observa é que em asininos a sua freqüência de ocorrência e volume médio são bem menores do que as médias registradas para garanhōes, onde o volume de gel pode representar mais da metade do volume total ejaculado ${ }^{13}$. Vários autores identificaram a presença de gel no ejaculado ${ }^{1.2 .15,20.28}$ sendo que os resultados são bastante variáveis, com ocorrência limitada aos jumentos mais velhos ${ }^{20}$. No presente trabalho, entretanto, a menor incidência correspondeu justamente ao jumento mais velho, com 9 anos, dados, portanto, ainda inconclusivos. $\mathrm{O}$ que merece ser salientado porém, é que apesar de o volume total do ejaculado de asininos ser menor do que o de equinos, a porção rica em espermatozóides é semelhante para ambas as espécies, sendo que a variação total se deve apenas a alterações no volume de gel.

Ao se avaliar os resultados da motilidade espermática, observa-se que a variação entre as amostras foi muito pequena, tendo sido menores as médias do jumento mais jovem $(F)$ e do jumento $\mathrm{D}$, cujo quadro espermático era de menor qualidade como um todo. Os resultados de motilidade total assemelham-se àqueles encontrados para garanhões ${ }^{12,26,31,33}$ porém, no que se refere à motilidade progressiva (retilínea $\mathrm{e}$ circular aberta), os nossos resultados foram superiores aos de equinos $3,23,34,38,39$. Ao se confrontar os dados com aqueles publicados para jumentos $1.2,7.15 .26,20,42$ houve uma grande semelhança tanto para motilidade total como progressiva, apesar da diversidade de condições experimentais.

No que se refere ao vigor espermático, a média geral obtida no presente estudo foi ligeiramente superior àquelas encontradas para asinino ${ }^{18,30} \mathrm{e}$ garanhōes ${ }^{31}$. Isso, na opinião deste úlltimo autor, indica amostras de elevada qualidade. as quais poderiam obter ótimos índices de congelabilidade.

Os resultados de concentração espermática e número total de espermatozóides por ejaculado são superiores àqueles encontrados para garanhões s.23,26,31,32, $34,38,39$ e semelhantes ou também superiores aos apresentados para jumentos 2.7.15,18,20,24,28,30,42 
Esta diferença entre asininos e equinos também foi observada por outros autores ${ }^{71,2 k}$, os qualis concluíram que al produção de espermatozóides de jumentos é bem maior do que a de garanhōes. Em programas de inseminação artificial isso refletiria em um maior número de doses por ejaculado para o reprodutor asinino.

Para a classificação das formas espermáticas adotamos o sistema proposto por BLOM", do mesmo modo que outros autores ${ }^{1.14,18,31} \mathrm{e}$, para aumentar as possibilidades de discussão ainda agrupamos as anomalias de acordo com sua localização 27.37 .40 . Considerando-se a poreentagem média de total de defeitos por ejaculado, os valores encontrados estão dentro dos limites aceitáveis para equinos ${ }^{6,25}$, não diferindo muito daqueles apresentados para jumentos ${ }^{1.212 \%}$. Ao dividirmos as patologias em defeitos maiores e menores, obtivemos médias que se apresentam em posição intermediária entre as de lal. WISHY ${ }^{14}$ e ARRUIDA et al.' c coincidentes com als de HENRY et al. ${ }^{18}$. Individualmente. predominaram as anoma- lias de cauda. na maioria dobradas e enroladas, o que concorda com relatos prévios ${ }^{20.29}$. Em seguida, aparecem alterações de cabeça, com predomínio de defeitos menores, tais como cabeças estreitas, pecjuenas, normais ou gigantes. $O$ jumento mais jovem (F) foi o que apresentou níveis mais elevados de patologia de cabeça, incluindo defeitos maiores, o que pode refletir un estado de atividade espermatogênica aquém de sua plenitude fisiológica, em função da idade. A terceira maior média refere-se às gotas citoplasmáticas distais e proximais. cujos valores são bem maiores do que os citados na literaturat 2019). apesar de não comprometerem a qualidade das amostras. estando dentro de limites aceitáveis. Notou-se lambém a presença de um grande número de células com implantação abaxial nas amostras analisadas, o que também foi observado por EL WISHY'4 Acreditamos que, assim como para equinos ${ }^{211.37}$. a presença de espermatozóides com implantação abaxial no sêmen de jumentos seja normal, ainda que não se tenha definido limites de tolerância.

\section{SLMMARY}

Seminal characteristics of six donkeys of the "Pega" breed, ranging from 3109 years of age were studied. Between October 1988 and April 1989, seventy (70) ejaculates were collected by using artificial vagina, twice a week. The following averatre values. lor the parameters studied were found: lotal volume $(59.88 \pm 15.54 \mathrm{ml})$. gel volume $(8.19 \pm 4.07 \mathrm{ml})$. total spermatozoal motility $(81.93 \pm 6.27 \%)$. progressive motility $(72.86 \pm 7.20 \%)$. sperm vigor $(4.63 \pm 0.49)$, sperm concentration $\left(444,11 \pm 182,72 \times 10^{\%} / \mathrm{mm}^{3}\right)$. total sperm number per ejaculate $\left(24.88 \pm 9.59 \times 10^{\prime \prime}\right)$. total abnormal celss $(15.60 \pm 3.62 \%)$, major delects $(8.55 \pm 2.14 \%)$ and minor defects $(7.04 \pm$ $2.56 \%$ ). These results were quite similar to that reported in the literatture for donkeys. Compared with the values lound by other authors for stallions. both species are very similar. differing only in some aspects. The total volume of jackass ejaculate was inferior, however. since the gel occurrence was much lower than in stallions. the "sperm-rich" fraction volume was approximately the same. The sperm concentration and total sperm number per ejaculate were higher for asses. Furthermore. the parameters of spermatozoal motility and vigor demonstrated that jackass semen was. almost al ways, of superior yuality. In artificial inseminatton programmes, these characteristics would indicate the possibility of higher number of individual-doses per ejaculate and better freezability levels.

UNITERMS: Reproduction: Donkeys; Semen: Morphology

\section{REFERÊNCIAS BIBIJOGRÁFICAS}

01-ARRUDA, R.P.; VIEIRA, R.C.; BARBOSA, R.T.; MANZANO, A. Congelação do sêmen de jumentos: características reprodutivas de um doador. Rev. bras. Reprod. Anim., p. 215, 1989. Suplemento 1.

02-BECK, E. Einfuhkung der Kunstlichen Besamung in der Maulticrzucht Marokkos. Tierarztl. Umsch., v.37. p.21-4. 1982.

03-BIELANSKI, W. Characteristics of the semen of stallions. Macro and microscopic investigations with estimation of fertility. Men. Acad. Pol. Sci. Ser.B. v.16, p.1-59, 1951 apud Anim. Bred. Abst. v.19, n.4, p.429-30, 1951. (resumo)

()4-BIFI.ANSKI, W. The evaluation of stallion semen in aspects of fertility control and its use in artificial insemination. J. Reprod. Fertil., p.19-24, 1975. Supplement 2.3.

05-BIELANSKI, W. Spermatozoa morphology in the stallion. Bihlphy Reprod., v.38, p.5()2-95, 1981. 
(06-BIELANSKI, W.; DUDEK, I:.; BITTMAR, A.; KOSINIAK, K. Some characteristics of common abnormal forms of spermatozoa in highly fertile stallions.J. Reprod. Fertil., p.21-6.1982. Supplement. 32.

07-BIELANSKI, W .: WIER\%OBOWSKI.S. Some properties of the output of semen of jacks. Acta Biol. Cracov. $S$. Zool., v.5, p.117-24, 1962.

()X-BL,OM, E. Interpretation of spermatic citology in bulls. Fertil. \& Steril, v.1, p. 223-8, 1950).

(0)-BI,OM, E. The ultrastructure of some characteristics sperm defects and a proposal for a new classification of hull spermiogram. Nord. Vet.-Med., v.25, p. 383 91,1973

10-IOOTT, H.M. Morphology of stallion spermatozoa. J. Reprod. Fertil.. p.41-6, 1975. Supplement 23.

1]-DOWSFTT, K.F.: OSBORNE. H.G.: PATTIE, W.A. Morphological characteristics of stallion spermatozoa. Theriogenology, v.22, p.463-72, 1984.

12-I)OWSFTT, K.F.; PATTIE, W.A. Characteristics and fertility of stallion semen. J. Reprod. Fertil., p. 1-8, 1982. Supplement 32.

13-LLL WISHY, A.B. Testicular and epididymal sperm reserves in the ass (Fquus asinus) and stallion (Equus caballus). Z. Tierzucht. Zuchstbiol., v.91, p. 33444. 1974

14-EL, WISHY, A.B. Morphology of epididymal spermatozoa in the ass (Equus asinus) and stallion (Equus cabalus). /.. 'Tierzuchtg. Zuchstbiol., v.92, p. 67-72, 1975.

15-GLAT/LI, P.: EL, HOUSSAIN, K.: TIBARY, A. Pferdeund Fselhengste der marokkanischen Landespferd und Maultierzucht. Erste Erghnisse aus deim Einsatz von Flussing-und Gefriersamen fur dir Maullicrproduktion. Berl. Münch. Tierarztl. Wschr., v. 94, p. 445-9, 1981.

16-IIANCOK, J.L. The morphology of boar spermatozoa. J. Roy. Microsc. Soc., v. 76, p. 84-97, 1957.

17-HARAZYMOWYC\%, J.; BALL. L.; SEIDEL, JUNIOR, G.E. Evaluation of hovine spermatozoal morphologic features after staining or fixation. Amer. J. vet. Res., v.37. p.1053-7, 1976.

I8-HENRY, M.; GASTAl. E.L.: MEIRA. C.; I)IAZ. A.P. Características do sêmen de jumentos da raça nordestina. In: CONGRESSO BRASILIEIRO DE REPROIJUÇÃO ANIMAL, 7., Belo Horizonte, 1987. Programa. Colégio Brasileirn de Reprodução Animal, 1987. p.72.
19-KENNEY, R.M.; KINGSTON, R.S.: RAJAMANNAN, A.H.J. Stallion semen characteristics for predicting fertility. In: AMERICAN ASSOCIATION EQUINE PRACTITIONER. Proceedings. 1971. p. 53-66.

20-KREUCHAUF, A. Zum fortpflanzungeschahen beim Esel (Equus asinus). Munchen, 1983. (Inaugural Dissertation Zur Erlangung der tiermedizinischen Doklorwurde) - Tierarallichen Fakultat der Ludwing - Maximilians, Universitat Munchen.

2I-KREUCHAUF, A. Reproduclive phisiology in the jackass. Anim. Res. Develop., v.20, p.51-78, 1984.

22-KUHI.MANN, J. Samenbiologische, biochemische und cryotechnische Untersuchungen an Fselsperma. Hannover, 1987. (Inaugural Dissertation /ur Erlangung der tiermedinischen Doktorwurde) - Tierarłtlichen Hochschule Hannover.

23-MAGISTRINI, M.; CHANTELOUBE, PH.: PALMER. E. Influence of season and frequency of ejaculation on production of stallion semen for freezing. J. Reprod. Fertil.. p. 127-33, 1987. Supplement 35.

24-MANN, T.; MINOTAKIS, C.S.; POL,GE, C. Semen composition and metabolism in the stallion and jackass. J. Reprod. Fertil., v. 5, p. 109-22, 196.3.

25-MIES FIL,H(), A. Reprodução dos animais e inseminação artificial. 6.ed. Porto Alegre, Sulina, 1987.

26-NISHIKAWA. Y.; WAIIDE. Y. On artificial insemination in the horse. $V$ - On the properties of semen and the factors affecting them. Bull. Nat. Inst. Agric. Sci. Ser G.. v. 1.p.13-28. 1951 apud Anim. Breed. Alst.. v.20, p.14, 1952. (resumo)

27-NISHIKAWA, Y.: WAIDE, Y. Studies on reproduction in asses. VI. On the method of eollection and the properties of semen. Bull. Nat. Inst. Agric. Sci. Ser. (.., v. 1, p. 37-45. 1951 apud Anim. Breed. Abst., v.20, p.14, 1952. (resumo)

28-NISHIKAWA, Y.; WAIDE, Y.; ONUMA, II. SIudies on artificial insemination in the horse. VI - Morphological studies on horse spermatozoa. Bull. Nat. Inst. Agric. Sci. Ser. (G. v. 1. p.29-36. 1951 apud Anim. Breed. Abst.. v.20, p.15, 1952. (resumo)

29-NISHIKAWA, Y.: WAIDE, Y.; ONUMA. H. Studies on reproduction in asses. VII. Morphological studies of semen. Bull. Nat. Inst. Agric. Sci. Ser. (i., v. 1. p. $47-$ 52, 1951 apud Anim. Breed. Abst., v.20, p. 15. 1952. (resumo)

30-OCES, N.C.: OSTROWSKY. J.E.B.; BÁl.OCCO, S.M. Producción de mulares por inseminación artificial. II. Exame microscopico, processamiento y uso de semen 
refrigerado de asno. Vet. Arg., v.1, p.236-45, 1984.

3 l-PAPA, F.O. Contribuição ao estudo de sêmen congelado de equinos: modificaçōes metodológicas para o congelamento e inseminaçăo artificial. Botucatu, 1987. Tese (Livre Docência) - Faculdade de Medicina Veterinária e Zootecnia, Universidade Estadual Paulista.

32-PATTIE, W.A.; DOWSETT, K.F. The repeatability of seminal characteristics of stallions.J. Reprod. Fertil., p.9-13, 1982. Supplement 32.

33-PICKETT, B.W.; FAUKER, L.C.; SEIDEL JUNIOR, G.E.; BERNDTSON, W.E; VOSS, J.L. Reproductive physiology of the stallion. VI. Seminal and behavioral characteristics. J. anim. Sci., v.43, p.617-25, 1976.

34-PICKETT, B.W.; FAULKNER, L.C.; SUTHERLAND, T.M. Effect of monch and stallion on seminal characteristics and sexual behavior. J. anim. Sci., v.31, p.713-28, 1970 .

35-PICKETT, B.W.; SQUIRES, E.L.; MCKINNON, A.O. Procedures for collection, evaluation and utilization of stallion semen for artificial insemination. Fort Collins, Anim. Reprod. Lab., Colorado State University, 1987.

36-ROUSSET, H.: CHANTELOUBE. P.H.; MAGISTRINI, M.; PALMER. E. Assessment of fertility and semen evaluations of stallions. J. Reprod. Fertil., p.25-31, 1987. Supplement 35.
37-SQUIRES, E.L.; AMANN, R.P.: PICKETT, B.W.; BERNDTSON, W.E.; SHIDELER, R.K. VOSS, J.L. Effect of fenbendazole on reproductive function in stallions. Theriogenology, v.9, p.447-55, 1978.

38-SQUIRES, E.L.; PICKETT, B.W.; AMANN, R.P. Effect of sucessive ejaculation on stallion seminal characteristics. J. Reprod. Fertil., p.7-12, 1979. Supplement 27.

39-VAN DER HOLST, W. A study of the morphology of stallion semen during the breeding and non-breeding seasons. J. Reprod. Fertil.. p.87-9, 1975. Supplement 23.

40-VOSS, J.L.; PICKETT, B.W.; SQUIRES, E.L. Slallion spermatozoal morphology motility and its relationships to fertility. J. Amer. Vet. Med. Ass., v.173, p.287-9, 1981.

41-WALTON, A. A flow orientation as a possible explanation of "wave motion" and "rheotaxis" of spermatozoa. J. exp. Biol.. v.29, p.520-31, 1952.

42-ZALCMANN, A.A. Characteristics of ass sperm. Dokl. Akad. Selsjkohoz Nauk., v.7, p.37-8, 1940 apud BIELANSKI; WIERZBOWSKI ${ }^{7}$, 1962. p.123.

Trabalho recebido para publicação em: 10/08/92 Aprovado para publicação em: 15/12/92 